\title{
Effects of cytokine-suppressive anti-inflammatory drugs on inflammatory activation in ex vivo human and ovine fetal membranes
}

\author{
Lisa F Stinson, Demelza J Ireland, Matthew W Kemp, Matthew S Payne, Sarah J Stock, \\ John P Newnham and Jeffrey A Keelan \\ School of Women's and Infants' Health, King Edward Memorial Hospital, The University of Western Australia, \\ 374 Bagot Road, Subiaco, Perth, Western Australia 6008, Australia
}

Correspondence should be addressed to J A Keelan; Email: jeff.keelan@uwa.edu.au

\begin{abstract}
Intrauterine infection and inflammation are responsible for the majority of early ( $<32$ weeks) spontaneous preterm births (PTBs). Anti-inflammatory agents, delivered intra-amniotically together with antibiotics, may be an effective strategy for preventing PTB. In this study, the effects of four cytokine-suppressive anti-inflammatory drugs (CSAIDs: $N$-acetyl cysteine (NAC), SB239063, TPCA-1 and NEMO binding domain inhibitor (NBDI)) were assessed on human and ovine gestational membrane inflammation. Full-thickness membranes were collected from healthy, term, human placentas delivered by Caesarean section $(n=5)$. Using a Transwell model, they were stimulated ex vivo with $\gamma$-irradiation-killed Escherichia coli applied to the amniotic face. Membranes from near-term, ovine placentas were stimulated in utero with lipopolysaccharide, Ureaplasma parvum or saline control and subjected to explant culture. The effects of treatment with CSAIDs or vehicle (1\% DMSO) on accumulation of PGE $_{2}$ and cytokines (human interleukin 6 (IL6), IL10 and TNF $\alpha$; ovine IL8 (oIL8)) were assessed in conditioned media at various time points (3-20 h). In human membranes, the IKK $\beta$ inhibitor TPCA-1 (7 $\mu$ M) and p38 MAPK inhibitor SB239063 $(20 \mu \mathrm{M})$ administered to the amniotic compartment were the most effective in inhibiting accumulation of cytokines and $\mathrm{PGE}_{2}$ in the fetal compartment. NAC (10 mM) inhibited accumulation of PGE 2 and IL10 only; NBDI $(10 \mu \mathrm{M})$ had no significant effect. In addition to the fetal compartment, SB239063 also exerted consistent and significant inhibitory effects in the maternal compartment. TPCA-1 and SB239063 suppressed olL8 production, while all CSAIDs tested suppressed ovine $\mathrm{PGE}_{2}$ production. These results support the further investigation of intra-amniotically delivered CSAIDs for the prevention of inflammation-mediated PTB.
\end{abstract}

Reproduction (2014) 147 313-320

\section{Introduction}

Preterm birth (PTB) remains a persistent obstetric challenge associated with significantly increased risk of neonatal mortality as well as short- and long-term morbidities (Goldenberg et al. 2008). The worldwide PTB rate is around $9.6 \%$, with rates typically lower in developed countries (5-8\%) and higher in developing nations (8-18\%) (Lawn et al. 2010). In addition to its impact on individuals and their families, PTB carries a substantial economic cost, estimated to be $\sim \$ 26$ billion annually in the USA in 2005 (Behrman \& Stith Butler 2007). Intrauterine infection and associated inflammation (most frequently diagnosed as presence of histological chorioamnionitis) have been identified as a cause of $30-40 \%$ of all spontaneous PTB (sPTB). Up to $70 \%$ of very early sPTBs ( $<32$ weeks gestation) are due to intrauterine infection-inflammation (Goldenberg et al. 2008).
Prophylactic antibiotic therapy has been extensively studied in the context of PTB prevention, with mixed results. While some studies have shown that administration of antibiotics (e.g. clindamycin) to high-risk women early in pregnancy $(<20$ weeks gestation) can have positive benefits in terms of reduced PTB rates and improved perinatal outcomes (Lamont et al. 2011), the majority of trials have failed to show significant benefits (Barros et al. 2010) and in some studies have even been shown to be harmful. The reasons for this are several fold and include issues related to participant selection and antibiotic efficacy, tissue biodistribution and microbial resistance (Keelan 2011). In addition, bactericidal antibiotics cause bacterial lysis and release of endotoxins, further activating the innate immune system and promoting the release of prostaglandins that may actually stimulate the onset of labour (Dofferhoff et al. 1991, Hurley 1995, Holzheimer 2001). 
We and others have proposed that a combined antiinflammatory/antibiotic approach may be more effective than antibiotics alone in treating intrauterine infectioninflammation, prolonging pregnancy and preventing fetal exposure to an inflammatory environment (Keelan 2011, Grigsby et al. 2012). Our present focus is on the intra-amniotic administration of anti-inflammatory/ antimicrobial agents in order to maximise therapeutic efficacy at the site of infection/inflammation, while minimising the risks of undesirable side effects through the reduction of unintended maternal or fetal exposure. Most of the literature on anti-inflammatory drugs in PTB has focussed on non-steroidal anti-inflammatory drugs (NSAIDs) - prostaglandin synthesis inhibitors that have widespread applications but which have been associated with significant fetal side effects (Kaplan et al. 1994, Nakhai-Pour et al. 2011). On the other hand, cytokinesuppressive anti-inflammatory drugs (CSAIDs) work by interfering with inflammatory signalling cascades and are therefore able to specifically block infection-mediated inflammation without some of the deleterious side effects of NSAIDs (Lee et al. 1989, Keelan 2011). CSAIDs have been shown to block inflammation in a variety of animal models of chronic inflammation (Underwood et al. 2000, Ward et al. 2001, Buhimschi et al. 2003, Jimi et al. 2004, di Meglio et al. 2005) as well as in human fetal membranes (Lappas et al. 2003, De Silva et al. 2010).

In this study, we evaluated four CSAIDs that target two key signalling pathways known to be involved in inflammatory activation of fetal membranes: nuclear

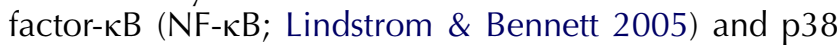
MAPK (Lappas et al. 2007). The CSAIDs were as follows: i) NEMO binding domain inhibitor (NBDI), ii) $\mathrm{N}$-acetyl cysteine (NAC), iii) TPCA-1 ((5-( $p$-fluorophenyl)-2ureido) thiophene-3-carboxamide) and iv) SB239063 (trans-1-(4-hydroxycyclohexyl)-4-(4-fluorophenyl)5-(2-(methoxy)pyrim-idin-4-yl)imidazole). An intraamniotic model of drug delivery was employed, as this approach allows for the targeting of gestational membranes and tissues (the key sites with respect to intra-amniotic infection-driven PTB) with minimal risk of unintended maternal immune modulation.

This study aimed to assess and compare the antiinflammatory efficacies of the four chosen CSAIDs on human and ovine gestational membranes using ex vivo models of intra-amniotic drug administration to assess their ability to inhibit inflammatory activation in both the amniotic and decidual faces of the gestational membranes.

\section{Subjects and methods \\ CSAIDs}

The concentrations of CSAIDs were as follows: NAC (Enzo Life Sciences, New York, NY, USA) 10 mM, NBDI (China Peptides, Shanghai, China) $10 \mu \mathrm{M}$, TPCA-1 (Merck Millipore, Darmstadt,
Germany) $7 \mu \mathrm{M}$ and SB239063 (Alexis Biochemicals, Lausen, Switzerland) $20 \mu \mathrm{M}$. Doses of CSAIDs were based on pilot studies or published data on in vitro efficacy (Underwood et al. 2000, Barone et al. 2001, Ward et al. 2001, Ju et al. 2002, Lappas et al. 2003, Jimi et al. 2004, di Meglio et al. 2005, Tas et al. 2006, Shahin et al. 2009, De Silva et al. 2010, Grassia et al. 2010).

\section{Human membrane Transwell model}

Full-thickness gestational membranes were collected from healthy, human, term placentas (38-40 weeks of gestation) delivered by Caesarean section $(n=5)$ with the approval of the local institutional Human Research Ethics Committee. Membranes were secured over $27 \mathrm{~mm}$ Transwell inserts (Corning, Inc., Lindfield, NSW, Australia) and placed in six-well culture plates containing serum-free culture media (DMEM/Ham's Nutrient Mixture F-12, phenol red-free, supplemented with 15 mM HEPES, pH 7.3 (Sigma-Aldrich Co.), 0.5\% endotoxin/ fatty acid-free BSA (Bovogen Biologicals Pty Ltd, East Keilor, VIC, Australia) and $4 \mu \mathrm{g} / \mathrm{ml}$ azithromycin (Pfizer, New York, NY, USA)). The maternal/decidual compartment contained $3 \mathrm{ml}$ media, while the inner/amniotic compartment contained $2.5 \mathrm{ml}$. $\gamma$-irradiation-killed Escherichia coli $(10 \mu \mathrm{g} / \mathrm{ml})$ and fluorescent Spherobeads $(4060 \mathrm{~nm}, 0.1 \mathrm{mg} / \mathrm{ml}$; Spherotech, Inc., Lake Forrest, IL, USA) were added to the inner compartment, followed by CSAIDs in $1 \%$ DMSO or vehicle ( $1 \%$ DMSO control) at $t=0 \mathrm{~h}$. Membranes were incubated for 20 h at $37{ }^{\circ} \mathrm{C}$ in $5 \% \mathrm{CO}_{2} / 95 \%$ air. Samples of conditioned media $(100 \mu \mathrm{l})$ were taken from the fetal and maternal compartments at 0,3 and $9 \mathrm{~h}$ and a final $1 \mathrm{ml}$ sample was taken at $20 \mathrm{~h}$. Structural integrity of the membranes was monitored by the passage of Spherobeads between inner/amniotic compartment and maternal/decidual compartments. Spherobead concentrations were measured in the fetal and maternal compartments by fluorescence using an FLx 800 plate fluorometer (BioTek Instruments, Inc., Winooski, VT, USA) at excitation 585/10 nm and emission 620/15 nm. Analysis of samples from both compartments of the Transwells showed that all membranes were intact with $>99 \%$ of Spherobeads retained within the fetal compartments and no significant fluorescence detected in the maternal compartments of any of the Transwells.

\section{Ovine membrane explant studies}

Animal studies were performed on pregnant Merino sheep (Ovis aries) in Western Australia with the approval of the University of Western Australia's Animal Ethics Committee $(\mathrm{RA} / 3 / 100 / 1098)$. The sheep in this study received intraamniotic injections at $117 \pm 2$ days gestational age (GA) of saline (2 ml, $n=2$ ), lipopolysaccharide (LPS; O55:B5; SigmaAldrich; $10 \mathrm{mg}$ in $2 \mathrm{ml}$ saline, $n=4)$ or Ureaplasma parvum serovar $3\left(10^{7}\right.$ colour change units in $2 \mathrm{ml}$ saline, $\left.n=4\right) 7$ days prior to delivery. All fetuses were surgically delivered at $124 \pm 2$ days GA (term $=150$ days) for necropsy. Fetal membranes were excised at this time and transported to the laboratory in media for explant culture. Explants were prepared from each set of membranes ( $8 \mathrm{~mm}$ discs), with three discs placed per well in 12-well plates and incubated in serum-free culture media at $37^{\circ} \mathrm{C} / 5 \% \mathrm{CO}_{2} .95 \%$ air. Treatment with the 
CSAIDs or vehicle (DMSO, 1\%) was carried out for $14 \mathrm{~h}$ before the explants were removed, the media were stored at $-80^{\circ} \mathrm{C}$ for later analysis and the tissues were air-dried overnight and weighed for normalisation.

\section{Measurement of cytokine and $P G E_{2}$ concentrations}

Accumulation of cytokines (human interleukin 10 (IL10), IL6 and TNF $\alpha$; ovine IL8 (olL8)) and $\mathrm{PGE}_{2}$ was measured in conditioned fetal and maternal media for the human Transwell study and from explant conditioned media for the ovine studies. Human IL10 and TNF $\alpha$ were measured by multiplex assay (Merck Millipore, Darmstadt, Germany) on a MAGPIX Instrument (Luminex Corp., Austin, TX, USA) as per the manufacturer's instructions. Human IL6 was measured using an ELISA Development Kit (PeproTech, Rocky Hill, NJ, USA) according to the recommended protocol. $\mathrm{PGE}_{2}$ was measured by prostaglandin $\mathrm{E}_{2}$ EIA Kit - monoclonal (Cayman Chemical Company, Ann Arbor, MI, USA) as per the manufacturer's instructions. olL8 was measured by in-house ELISA calibrated against recombinant olL8 from Protein Express, Inc. (Cincinnati, $\mathrm{OH}$, USA) using a mouse anti-sheep IL8 monoclonal capture antibody (MCA1660: $5 \mu \mathrm{g} / \mathrm{ml}$ overnight) and a rabbit anti-sheep IL8 polyclonal antibody (AHP425: 1:1000 2 h) from AbD Serotec (Raleigh, NC, USA). Detection and quantitation involved an anti-rabbit IgG-HRPO conjugate $(1: 10001 \mathrm{~h})$ and TMB substrate. The limits of detection of the IL10, TNF $\alpha$, IL6, $\mathrm{PGE}_{2}$ and olL8 assays were $<3.2,<3.2,100,7$ and $33 \mathrm{pg} / \mathrm{ml}$ respectively. Media samples were diluted 1:10 for the IL6 assay, 1:5 for the $\mathrm{PGE}_{2}$ assay, 1:2 for the IL8 assay and were undiluted for the IL10 and TNF $\alpha$ assays.

\section{Statistical analysis}

To adjust for variable baseline expression between membranes from different placentas, the concentrations of cytokines and $\mathrm{PGE}_{2}$ within the conditioned media from each Transwell were expressed as a percentage of the sum of concentrations from all six Transwells from each set of membranes. The production data from the sheep explants were similarly normalised prior to statistical analysis. Data are shown as median \pm interquartile range $(\mathrm{IQR})$ or mean \pm s.E.M. Unless stated otherwise, all statistical significance was assessed by one-way ANOVA followed by Dunnett's t-test post-hoc analyses (Prism, GraphPad Software, Inc., La Jolla, CA, USA). Non-parametric data were log transformed prior to analysis. A $P$ value of $<0.05$ was considered significant. For analysis of basal and stimulated cytokine production rates in human membranes, significance was assessed by Wilcoxon matched pairs test, both at each individual time point and overall.

\section{Results}

\section{Cytokine and prostaglandin production by stimulated human fetal membranes in the Transwell perfusion model}

Figure 1 shows the baseline production of cytokines and $\mathrm{PGE}_{2}$ over time $(3,9$ and $20 \mathrm{~h})$ in vehicle (DMSO) or
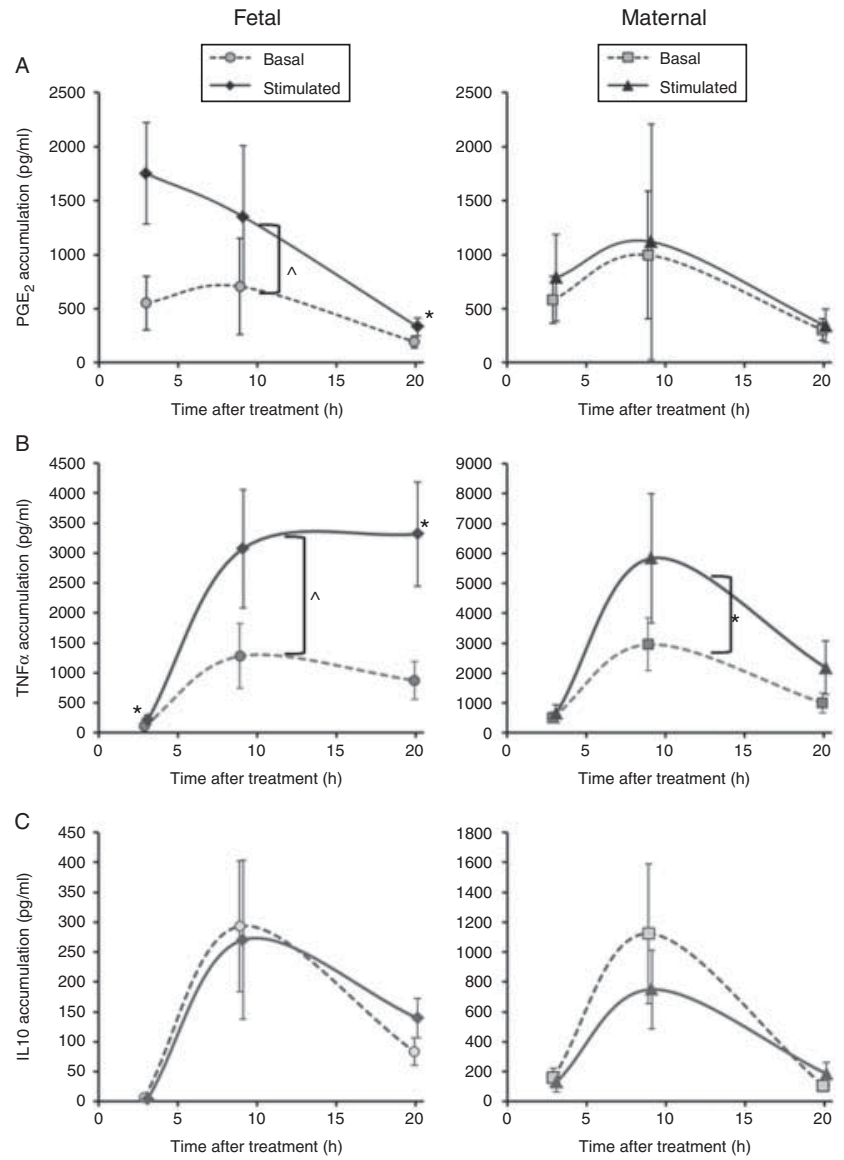

Figure 1 Time-dependent changes in accumulation of $(A) \mathrm{PGE}_{2}$, (B) TNF $\alpha$ and (C) IL10 in the conditioned media from the maternal and fetal compartments of human fetal membranes in the Transwell model following exposure to vehicle (basal) or $10 \mu \mathrm{g} / \mathrm{ml} \gamma$-irradiation killed Escherichia coli (stimulated) at the amniotic face. Data shown are concentration $(\mathrm{pg} / \mathrm{ml})$, mean \pm s.E.M. ( $n=5$ sets of membranes). ${ }^{*} P<0.05$ and ${ }^{\wedge} P<0.001$ basal vs stimulated by Wilcoxon matched pairs test.

E. coli-stimulated human Transwells. Basal $\mathrm{PGE}_{2}$ accumulation in the fetal compartment increased modestly from 3 to $9 \mathrm{~h}$, then declined at $20 \mathrm{~h}$ (Fig. 1A); mean concentrations at $9 \mathrm{~h}$ were $\sim 700 \mathrm{pg} / \mathrm{ml}$. Basal $\mathrm{PGE}_{2}$ levels were a little higher in the maternal compartment, peaking at $\sim 1000 \mathrm{pg} / \mathrm{ml}$ at $9 \mathrm{~h}$ before declining by $20 \mathrm{~h}$. With bacterial stimulation, however, levels in the fetal compartment rose markedly at $3 \mathrm{~h}$ to $>1700 \mathrm{pg} / \mathrm{ml}$ and then declined thereafter, whereas in the maternal compartment no evidence of stimulation was observed. The effect of stimulation in the fetal compartment was significant at $20 \mathrm{~h}(P<0.05)$ and over all time points $(P<0.001)$. TNF $\alpha$ accumulation in the fetal compartment under basal conditions also peaked at the 9-h incubation period, reaching $\sim 1300 \mathrm{pg} / \mathrm{ml}$ (Fig. 1B). Similarly, maternal basal TNF $\alpha$ concentrations also peaked at $9 \mathrm{~h}(\sim 2900 \mathrm{pg} / \mathrm{ml})$, then declined to $\sim 1000 \mathrm{pg} / \mathrm{ml}$ at $20 \mathrm{~h}$. With bacterial stimulation, concentrations of TNF $\alpha$ in the fetal compartment were 
significantly (two- to threefold) elevated at 3 and $20 \mathrm{~h}$ $(P<0.05$; overall significance: $P<0.001)$, with a significant stimulation also seen in the maternal compartment $(P<0.05$ overall). Fetal IL10 levels were low or undetectable at $3 \mathrm{~h}$, but rose to concentrations of $\sim 300 \mathrm{pg} / \mathrm{ml}$ at $9 \mathrm{~h}$ before declining at $20 \mathrm{~h}$ (Fig. 1C). Maternal basal IL10 levels were significantly higher than fetal levels at $3 \mathrm{~h}(P<0.01)$ and peaked at $1100 \mathrm{pg} / \mathrm{ml}$ at $9 \mathrm{~h}$, after which they progressively declined to $20 \mathrm{~h}$. Stimulation with $E$. coli failed to increase IL10 levels in either compartment.

\section{Anti-inflammatory effects of CSAIDs on human fetal membranes}

Treatment of $E$. coli-stimulated human gestational membranes at the amniotic face with NBDI had no significant effects on $\mathrm{PGE}_{2}$ accumulation in either compartment, although at the 9-h time point median maternal $\mathrm{PGE}_{2}$ levels were reduced by $\sim 50 \%$ (Fig. $2 \mathrm{~A}$ ). Treatment with NAC resulted in a non-significant $60 \%$ reduction in $\mathrm{PGE}_{2}$ accumulation relative to DMSO controls in the fetal compartment at $9 \mathrm{~h}$ and a smaller $(\sim 35 \%)$ reduction in the maternal compartment at $20 \mathrm{~h}$ (Fig. 2A); TPCA-1 resulted in significant $(\sim 70 \%$; $P<0.05)$ suppression of $\mathrm{PGE}_{2}$ accumulation in the fetal (but not maternal) compartment at 3, 9 and $20 \mathrm{~h}$ post treatment (Fig. 2A). SB239063 also significantly inhibited $\mathrm{PGE}_{2}$ accumulation at all time points in the fetal compartment (80-85\%; $P<0.05$ ), but unlike TPCA-1, it was also able to significantly reduce $\mathrm{PGE}_{2}$ levels in the maternal compartment ( $\sim 70$ and $87 \%$ at 9 and $20 \mathrm{~h}$; $P<0.05$ and $<0.001$ respectively).

Neither NBDI nor NAC significantly affected TNF $\alpha$ levels at any time point (Fig. 2B). TNF $\alpha$ accumulation in the fetal compartment was, however, markedly reduced by both TPCA-1 and SB239063, with significant reductions observed at $3 \mathrm{~h}(P<0.01)$, becoming more evident at 9 and $20 \mathrm{~h}(P<0.001)$. TPCA-1 significantly reduced maternal TNF $\alpha$ accumulation by $\sim 75 \%$ at $9 \mathrm{~h}$ $(P<0.05)$. SB239063 was again the most effective antiinflammatory agent in the maternal compartment with significant inhibitions of $\sim 95$ and $\sim 62 \%$ seen at 9 and 20 h respectively $(P<0.001)$.

NBDI had no effect on IL10 production in either compartment; however, IL10 accumulation was inhibited by the other CSAIDs at the 9 and $20 \mathrm{~h}$ time points (Fig. 2C). Within the fetal compartment, NAC, TPCA-1 and SB239063 resulted in significant reductions in IL10 production with effects at $20 \mathrm{~h}$ in the region of $85-92 \%$ $(P<0.001)$. The same three CSAIDs reduced IL10 accumulation in the maternal compartment, but the level of inhibition did not reach statistical significance due to large variability in the vehicle controls. The inhibitory effect of the anti-inflammatory agents tended to increase with time, although this trend was not statistically significant. The effect was most apparent for IL10 (Fig. 2C).
Inhibition of IL6 accumulation was assessed at $20 \mathrm{~h}$ only, due to insufficient media at the earlier time points. Once more, NBDI failed to exert significant effects. There was again a trend towards inhibition by NAC and TPCA-1 in the fetal compartment, although the degree of inhibition was more modest than that seen for the other cytokines (38-62\%; Fig. 2D). SB239063 was the most effective anti-inflammatory agent in both compartments and significantly inhibited IL6 accumulation at the 20-h time point $(P<0.05)$.

\section{Anti-inflammatory effects of CSAIDs on ovine fetal membrane explants}

The efficacies of the four CSAIDs were evaluated in fullthickness gestational membranes from near-term sheep. Explants were employed for the ovine studies as attempts to replicate the human Transwell study with ovine membranes were not successful. CSAID dosages and incubation times were based on the results from the human studies. Samples were initially assayed for olL $1 \beta$, IL8, IL10, TNF $\alpha, \mathrm{MCP}-1$ and $\mathrm{PGE}_{2}$; however, only concentrations of IL8 and $\mathrm{PGE}_{2}$ were above the detection limits of the assays employed and generated meaningful data.

Median $\mathrm{PGE}_{2}$ concentration in media from full-thickness gestational membrane explants from saline-treated sheep was $9.1 \mathrm{pg} / \mathrm{mg}$ tissue at $14 \mathrm{~h}$, while median olL8 levels were $53.0 \mathrm{pg} / \mathrm{mg}$ tissue. Membranes from sheep stimulated with LPS or $U$. parvum exhibited modest and variable increases in production of olL8 and $\mathrm{PGE}_{2}$ that did not reach statistical significance compared with saline-treated controls. Therefore, the data from all the three groups $(n=9$ sets of membranes) were analysed collectively.

In contrast to the human study, NBDI was as effective as the other CSAIDs at inhibiting $\mathrm{PGE}_{2}$ accumulation in ovine gestational membranes. $\mathrm{PGE}_{2}$ accumulation was significantly inhibited $(65-71 \% ; P<0.01)$ by all four CSAIDs compared with the DMSO vehicle-treated explants (Fig. 3A). However, NBDI and NAC had no effect on olL8 levels, and the effects of NAC treatment on olL8 levels were particularly variable. TPCA-1 significantly reduced olL8 accumulation by $80 \%(P<0.01)$, while SB239063 significantly reduced olL8 levels by $\sim 60 \%(P<0.01)$ (Fig. 3B).

\section{Discussion}

PTB remains a major obstetric issue throughout the world and is associated with significant perinatal morbidity and mortality and lifelong health and economic consequences. In 2010, 14.9 million preterm deliveries occurred worldwide, from which over 1 million infants died as a result of their prematurity (Blencowe et al. 2012). Despite decades of research on PTB aetiologies, few therapeutic options are available to women at risk of delivering preterm. Here, we have 

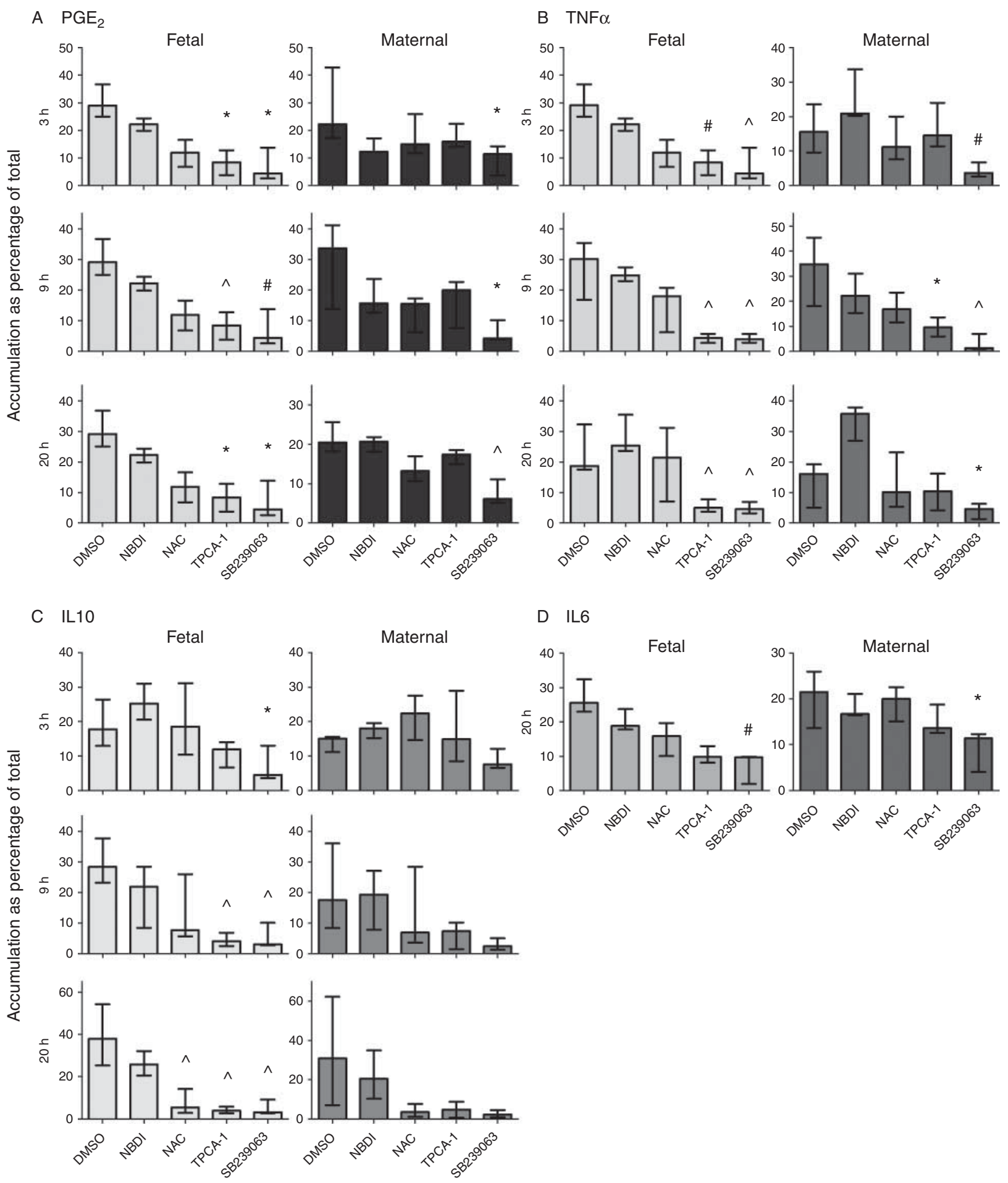

D IL6

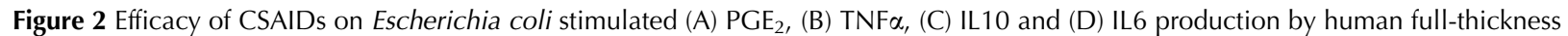
fetal membranes in an ex vivo Transwell perfusion model at 3-, 9- and 20-h culture. Data are median \pm IQR from $n=5$ placentas, normalised as a percentage of total analyte production per set of experiments. ${ }^{*} P<0.05,{ }^{\sharp} P<0.01$ and ${ }^{\wedge} P<0.001$ relative to vehicle (DMSO) control. Significance was assessed by two-tailed ANOVA after log-transformation of data.

investigated the ex vivo efficacy of a number of anti-inflammatory agents based on the hypothesis that intra-amniotic CSAID administration can provide a pharmacological strategy for the prevention of infection/ inflammation-mediated PTB. The CSAIDs selected for this study were as follows: i) NBDI, a cell-permeable peptide that spans the NF-KB essential modifier (NEMO) binding domain sequence (Madge \& May 2009), which 


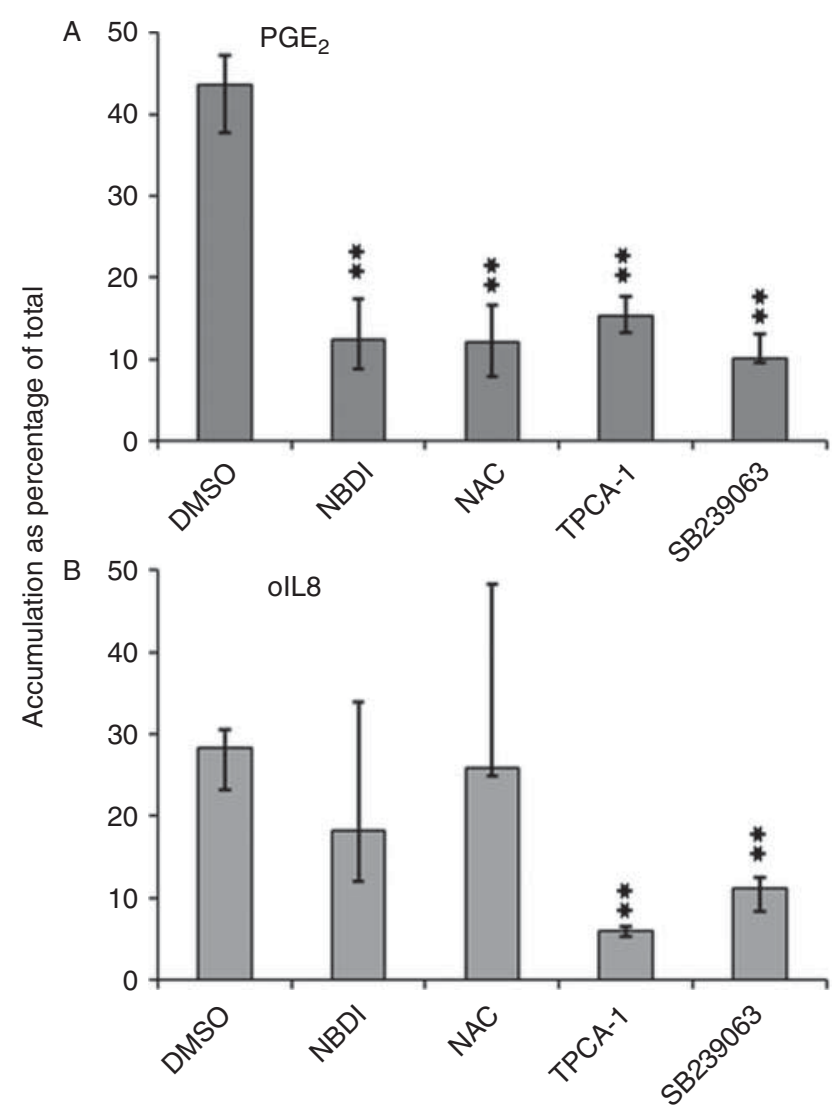

Figure 3 Effects of CSAIDs on (A) $\mathrm{PGE}_{2}$ and (B) IL8 accumulation in ovine fetal membranes $(n=9)$ over $14 \mathrm{~h}$ in explant culture. Data are expressed as a percentage of total accumulation for each set of explants (median $\pm \mathrm{IQR}) .{ }^{*} P<0.01$ relative to vehicle control (DMSO). Significance was assessed by two-tailed ANOVA after logtransformation of data.

has been shown to block inflammation effectively in in vivo animal models (Jimi et al. 2004, di Meglio et al. 2005, Tas et al. 2006, Grassia et al. 2010); ii) NAC, a powerful antioxidant and free radical scavenger that has been shown in a randomised controlled trial to reduce the rate of PTB when taken orally in women with a history of PTB and in whom bacterial vaginosis has recently been treated (Shahin et al. 2009); iii) TPCA-1, a selective IKK $\beta$ inhibitor (Podolin et al. 2005, Kondo et al. 2008) that is effective at inhibiting inflammation in vitro (Podolin et al. 2005, Sachse et al. 2011) and in vivo (Birrell et al. 2006) and iv) SB239063, a selective, potent and cell-permeable p38 MAPK inhibitor that has previously been shown to suppress inflammation in vivo (Underwood et al. 2000, Barone et al. 2001, Ward et al. 2001, Ju et al. 2002). They were studied in a human Transwell system to model the structural characteristics of intact gestational membranes and allow the assessment of efficacy of intra-amniotic anti-inflammatory drug delivery at both the maternal and fetal faces of the membranes. In parallel, in vivo stimulated ovine fetal membranes were also exposed to the CSAIDs to allow a comparison of efficacy between ovine and human tissues. DMSO vehicle was employed as previous studies in our laboratory have indicated that this solvent does not significantly alter inflammatory cytokine production by gestational tissues.

While all the four CSAIDs showed some degree of efficacy in both models, two were clearly superior: the IKK $\beta$ inhibitor TPCA1 and the p38 MAPK inhibitor SB239063. At the concentrations employed, both these compounds induced profound inhibitory effects on cytokine and prostaglandin accumulation in the fetal compartment of the Transwell model, with the MAPK inhibitor exerting more modest effects in the maternal compartment. The same degree of inhibition by these two CSAIDs was also seen in the ovine explant model, regardless of mode of stimulation. These findings support our hypothesis and provide rationale for the further investigation of these compounds in human gestational tissues derived from spontaneous preterm deliveries.

The central importance of NF- $\mathrm{KB}$ activation in the regulation of inflammatory gene expression is well recognised. We have previously shown that $5-7 \mu \mathrm{M}$ TPCA- 1 achieved $\sim 90 \%$ suppression of pro-inflammatory cytokine production and blocks nuclear translocation of p65/RelA in LPS-stimulated choriodecidual cells (De Silva et al. 2010). Until now, no studies have examined the effect of TPCA-1 in full-thickness gestational membranes, although it has been shown to be an effective inhibitor of the NF- $\kappa$ B pathway in a variety of other inflammatory models (Podolin et al. 2005, Birrell et al. 2006, Kondo et al. 2008, Du et al. 2012). Interestingly, in this study, the actions of TPCA- 1 were primarily restricted to the fetal (amniotic) compartment. This may reflect a lack of ability to penetrate the membrane barrier, restricting its actions to the amniotic epithelium, or may indicate that a higher dose is required to more completely block the transmembrane inflammatory signalling cascades. TPCA-1 also reduced $\mathrm{PGE}_{2}$ and IL8 production from ovine fetal membranes, confirming its effectiveness as an IKK $\beta$ inhibitor in the ovine species.

By contrast, the p38 MAPK inhibitor SB239063 was much more effective at inhibiting cytokine and prostaglandin accumulation at the maternal face, suggesting that it is either considerably more membrane permeable or has more profound effects on initial inflammatory signalling pathways. Its similar potency to TPCA- 1 in the ovine explant model would argue against the latter hypothesis. To date, no studies have characterised the expression and activity patterns of MAPKs during inflammation in human gestational membranes, although MAPKs are known to respond to infectious stimuli and regulate the production of pro-inflammatory cytokines (Underwood et al. 2000, Barone et al. 2001, Ward et al. 2001, Ju et al. 2002, Shoji et al. 2007). Regardless, our results highlighted SB239063 as a potentially effective anti-inflammatory agent useful for preventing inflammation-driven PTB. 
At the concentration used $(10 \mu \mathrm{M}), \mathrm{NBDI}$ was unable to inhibit production of any of the measured inflammatory markers in either compartment of the human Transwell model. We had selected a dose of $10 \mu \mathrm{M}$ of NBDI as this peptide has been successfully used at $0.1-1 \mu \mathrm{M}$ in a study of injury-induced inflammation in rats (Grassia et al. 2010). Surprisingly, it was notably more effective in the ovine explants at suppressing $\mathrm{PGE}_{2}$ accumulation. It remains to be determined whether a species difference in binding affinity or a relative insensitivity of the amnion membrane might explain these observations.

NAC, which exerts its effects through dampening of oxygen free radical reactions, has been shown to suppress NF- $\kappa B$ DNA binding activity in all the three layers of gestational membranes at $\geq 10 \mathrm{mM}$ (Lappas et al. 2003). In our study, NAC (10 mM) was not a particularly effective inhibitor of fetal membrane cytokine production but did appear to reduce $\mathrm{PGE}_{2}$ accumulation in the fetal side of the human Transwells. It also significantly reduced $\mathrm{PGE}_{2}$ levels in conditioned media from sheep membrane explants. NAC can directly inhibit prostaglandin biosynthesis via inhibition of the production of $\mathrm{PGH}_{2}$ by cyclooxygenases (De Flora et al. 2001), a reaction that involves a free radical step (Rouzer \& Marnett 2009), so its effects on prostaglandin inhibition are consistent with expectations. These findings add some weight to the evidence that NAC might be an effective antiinflammatory agent within the pregnant uterus (Lappas et al. 2003) and may be useful at preventing SPTB in some pregnancies (Shahin et al. 2009, Awad et al. 2011). The effectiveness of intra-amniotic delivery of NAC in vivo has not yet been explored.

The ovine membrane explant model used in this study employed membranes exposed in vivo to saline, LPS or $U$. parvum. This model has been developed over many years and is now extensively employed in obstetrics research (Kallapur et al. 2001, Moss et al. 2003, 2005). Unexpectedly, we did not observe a consistent and significant difference in $\mathrm{PGE}_{2}$ or IL8 production from control or stimulated membranes, although mean levels of both mediators were two- to threefold higher in the stimulated membranes compared with controls. The inter-animal variability might have been due to regional differences in levels of activation of membranes. Owing to the lack of significance, the data from all membranes were combined and hence we are unable to make conclusions regarding the efficacy of the CSAIDs with respect to different stimuli.

In conclusion, the results presented in this study identified TPCA-1 and SB239063 as CSAIDs of promise for pharmacological prevention of intra-amniotic inflammation. Further in vivo studies are justified to explore their ability to ameliorate the negative effects of intrauterine infection-driven inflammation. In combination with an effective antibiotic regimen, CSAIDs administered intra-amniotically may have significant clinical benefits in treating pregnancies at high risk of SPTB due to intrauterine infection-inflammation.

\section{Declaration of interest}

The authors declare that there is no conflict of interest that could be perceived as prejudicing the impartiality of the research reported.

\section{Funding}

This study was supported by the Women and Infants Research Foundation, WA, and the National Health and Medical Research Council (grant number APP1024467).

\section{Acknowledgements}

The assistance of the staff of the Large Animal Facility, The University of Western Australia, and our commercial sheep suppliers, Sara and Andrew Ritchie from Icon Agriculture in Darkan, Western Australia are gratefully acknowledged. The authors thank Prof. Boris Kramer and Suhas Kallipur for their assistance in harvesting the ovine fetal membranes. They also thank Dr Phillip Bird, University of Queensland, for the radiation-killed E. coli. They are also grateful to the staff at King Edward Memorial Hospital, Subiaco, Australia, for supporting this research and to the mothers who donated their placentas for the study.

\section{References}

Awad N, Khatib N, Ginsberg Y, Weiner Z, Maravi N, Thaler I, Ross MG, Itsokovitz-Eldor J \& Beloosesky R $2011 \quad \mathrm{~N}$-acetyl-cysteine (NAC) attenuates LPS-induced maternal and amniotic fluid oxidative stress and inflammatory responses in the preterm gestation. American Journal of Obstetrics and Gynecology 204 450.e15-450.e20. (doi:10.1016/j.ajog.2011.01.030)

Barone FC, Irving EA, Ray AM, Lee JC, Kassis S, Kumar S, Badger AM, White RF, McVey MJ, Legos JJ et al. 2001 SB 239063, a secondgeneration p38 mitogen-activated protein kinase inhibitor, reduces brain injury and neurological deficits in cerebral focal ischemia. Journal of Pharmacology and Experimental Therapeutics 296 312-321.

Barros FC, Bhutta ZA, Batra M, Hansen TN, Victora CG \& Rubens CE 2010 Global report on preterm birth and stillbirth (3 of 7): evidence for effectiveness of interventions. BMC Pregnancy and Childbirth 10 (Suppl 1) S3. (doi:10.1186/1471-2393-10-S1-S3)

Behrman RA \& Stith Butler A 2007 In Preterm Birth: Causes, Consequences and Prevention. Institute of Medicine (US) Committee on Understanding Premature Birth and Assuring Healthy Outcomes. Washington, DC: National Academies Press.

Birrell MA, Wong S, Hardaker EL, Catley MC, McCluskie K, Collins M, Haj-Yahia S \& Belvisi MG 2006 І $\kappa$ B kinase-2-independent and -dependent inflammation in airway disease models: relevance of IKK-2 inhibition to the clinic. Molecular Pharmacology 69 1791-1800. (doi:10.1124/mol.105.019521)

Blencowe H, Cousens S, Oestergaard MZ, Chou D, Moller AB, Narwal R, Adler A, Vera Garcia C, Rohde S, Say L et al. 2012 National, regional, and worldwide estimates of preterm birth rates in the year 2010 with time trends since 1990 for selected countries: a systematic analysis and implications. Lancet 379 2162-2172. (doi:10.1016/S0140-6736(12) 60820-4)

Buhimschi IA, Buhimschi CS \& Weiner CP 2003 Protective effect of $\mathrm{N}$-acetylcysteine against fetal death and preterm labor induced by maternal inflammation. American Journal of Obstetrics and Gynecology 188 203-208. (doi:10.1067/mob.2003.112)

De Flora S, Izzotti A, D'Agostini F \& Balansky RM 2001 Mechanisms of $\mathrm{N}$-acetylcysteine in the prevention of DNA damage and cancer, with special reference to smoking-related end-points. Carcinogenesis 22 999-1013. (doi:10.1093/carcin/22.7.999) 
De Silva D, Mitchell MD \& Keelan JA 2010 Inhibition of choriodecidual cytokine production and inflammatory gene expression by selective $\mathrm{I}-\kappa \mathrm{B}$ kinase (IKK) inhibitors. British Journal of Pharmacology $1601808-1822$. (doi:10.1111/j.1476-5381.2010.00839.x)

Dofferhoff AS, Nijland JH, de Vries-Hospers HG, Mulder PO, Weits J \& Bom VJ 1991 Effects of different types and combinations of antimicrobial agents on endotoxin release from gram-negative bacteria: an in-vitro and in-vivo study. Scandinavian Journal of Infectious Diseases 23 745-754. (doi:10.3109/00365549109024303)

Du Z, Whitt MA, Baumann J, Garner JM, Morton CL, Davidoff AM \& Pfeffer LM 2012 Inhibition of type I interferon-mediated antiviral action in human glioma cells by the IKK inhibitors BMS-345541 and TPCA-1. Journal of Interferon \& Cytokine Research 32 368-377. (doi:10.1089/ jir.2012.0002)

Goldenberg RL, Culhane JF, lams JD \& Romero R 2008 Epidemiology and causes of preterm birth. Lancet 371 75-84. (doi:10.1016/S01406736(08)60074-4)

Grassia G, Maddaluno M, Musilli C, De Stefano D, Carnuccio R, Di Lauro MV, Parratt CA, Kennedy S, Di Meglio P, lanaro A et al. 2010 The $I\{\kappa\} B$ kinase inhibitor nuclear factor- $\{\kappa\} B$ essential modulatorbinding domain peptide for inhibition of injury-induced neointimal formation. Arteriosclerosis, Thrombosis, and Vascular Biology $\mathbf{3 0}$ 2458-2466. (doi:10.1161/ATVBAHA.110.215467)

Grigsby PL, Novy MJ, Sadowsky DW, Morgan TK, Long M, Acosta E, Duffy LB \& Waites KB 2012 Maternal azithromycin therapy for Ureaplasma intraamniotic infection delays preterm delivery and reduces fetal lung injury in a primate model. American Journal of Obstetrics and Gynecology 207 475.e1-475.e14. (doi:10.1016/j.ajog.2012.10.871)

Holzheimer RG 2001 Antibiotic induced endotoxin release and clinical sepsis: a review. Journal of Chemotherapy 13 159-172. (doi:10.1179/ joc.2001.13.Supplement-2.159)

Hurley JC 1995 Antibiotic-induced release of endotoxin. A therapeutic paradox. Drug Safety 12 183-195. (doi:10.2165/00002018-199512030-00004)

Jimi E, Aoki K, Saito H, D'Acquisto F, May MJ, Nakamura I, Sudo T, Kojima T, Okamoto F, Fukushima $\mathbf{H}$ et al. 2004 Selective inhibition of NF- $\kappa$ B blocks osteoclastogenesis and prevents inflammatory bone destruction in vivo. Nature Medicine 10 617-624. (doi:10.1038/nm1054)

Ju H, Nerurkar S, Sauermelch CF, Olzinski AR, Mirabile R, Zimmerman D, Lee JC, Adams J, Sisko J, Berova M et al. 2002 Sustained activation of p38 mitogen-activated protein kinase contributes to the vascular response to injury. Journal of Pharmacology and Experimental Therapeutics 301 15-20. (doi:10.1124/jpet.301.1.15)

Kallapur SG, Willet KE, Jobe AH, Ikegami M \& Bachurski CJ 2001 Intraamniotic endotoxin: chorioamnionitis precedes lung maturation in preterm lambs. American Journal of Physiology. Lung Cellular and Molecular Physiology 280 L527-L536.

Kaplan BS, Restaino I, Raval DS, Gottlieb RP \& Bernstein J 1994 Renal failure in the neonate associated with in utero exposure to nonsteroidal anti-inflammatory agents. Pediatric Nephrology 8 700-704. (doi:10.1007/BF00869093)

Keelan JA 2011 Pharmacological inhibition of inflammatory pathways for the prevention of preterm birth. Journal of Reproductive Immunology $\mathbf{8 8}$ 176-184. (doi:10.1016/j.jri.2010.11.003)

Kondo Y, Fukuda K, Adachi T \& Nishida T 2008 Inhibition by a selective І $\mathrm{B}$ kinase-2 inhibitor of interleukin-1-induced collagen degradation by corneal fibroblasts in three-dimensional culture. Investigative Ophthalmology \& Visual Science 49 4850-4857. (doi:10.1167/iovs.08-1897)

Lamont RF, Nhan-Chang CL, Sobel JD, Workowski K, Conde-Agudelo A \& Romero R 2011 Treatment of abnormal vaginal flora in early pregnancy with clindamycin for the prevention of spontaneous preterm birth: a systematic review and metaanalysis. American Journal of Obstetrics and Gynecology 205 177-190. (doi:10.1016/j.ajog.2011.03.047)

Lappas M, Permezel M \& Rice GE $2003 \quad N$-acetyl-cysteine inhibits phospholipid metabolism, proinflammatory cytokine release, protease activity, and nuclear factor- $\mathrm{B}$ deoxyribonucleic acid-binding activity in human fetal membranes in vitro. Journal of Clinical Endocrinology and Metabolism 88 1723-1729. (doi:10.1210/jc.2002-021677)

Lappas M, Permezel M \& Rice GE 2007 Mitogen-activated protein kinase proteins regulate LPS-stimulated release of pro-inflammatory cytokines and prostaglandins from human gestational tissues. Placenta 28 936-945. (doi:10.1016/j.placenta.2007.02.009)
Lawn JE, Gravett MG, Nunes TM, Rubens CE \& Stanton C 2010 Global report on preterm birth and stillbirth (1 of 7): definitions, description of the burden and opportunities to improve data. BMC Pregnancy and Childbirth 10 (Suppl) S1. (doi:10.1186/1471-2393-10-S1-S1)

Lee JC, Votta B, Griswold DE \& Hanna N 1989 Inhibitory effect of SK\&F 86002 on monocyte IL-1 production. Agents and Actions 27 280-281. (doi:10.1007/BF01972797)

Lindstrom TM \& Bennett PR 2005 The role of nuclear factor $\kappa B$ in human labour. Reproduction 130 569-581. (doi:10.1530/rep.1.00197)

Madge LA \& May MJ 2009 Inhibiting proinflammatory NF- $\kappa$ B signaling using cell-penetrating NEMO binding domain peptides. Methods in Molecular Biology 512 209-232. (doi:10.1007/978-1-60327-530-9_11)

di Meglio P, Ianaro A \& Ghosh S 2005 Amelioration of acute inflammation by systemic administration of a cell-permeable peptide inhibitor of NF-кB activation. Arthritis and Rheumatism 52 951-958. (doi:10.1002/ art.20960)

Moss TJ, Nitsos I, Newnham JP, Ikegami M \& Jobe AH 2003 Chorioamnionitis induced by subchorionic endotoxin infusion in sheep. American Journal of Obstetrics and Gynecology 189 1771-1776. (doi:10.1016/ S0002-9378(03)00810-X)

Moss TJ, Nitsos I, Ikegami M, Jobe AH \& Newnham JP 2005 Experimental intrauterine Ureaplasma infection in sheep. American Journal of Obstetrics and Gynecology 192 1179-1186. (doi:10.1016/j.ajog.2004. 11.063)

Nakhai-Pour HR, Broy P, Sheehy O \& Berard A 2011 Use of nonaspirin nonsteroidal anti-inflammatory drugs during pregnancy and the risk of spontaneous abortion. CMAJ: Canadian Medical Association Journal 183 1713-1720. (doi:10.1503/cmaj.110454)

Podolin PL, Callahan JF, Bolognese BJ, Li YH, Carlson K, Davis TG, Mellor GW, Evans C \& Roshak AK 2005 Attenuation of murine collageninduced arthritis by a novel, potent, selective small molecule inhibitor of I $\mathrm{B}$ kinase 2, TPCA-1 (2-[(aminocarbonyl)amino]-5-(4-fluorophenyl)-3thiophenecarboxamide), occurs via reduction of proinflammatory cytokines and antigen-induced T cell proliferation. Journal of Pharmacology and Experimental Therapeutics 312 373-381. (doi:10.1124/jpet.104. 074484)

Rouzer CA \& Marnett LJ 2009 Cyclooxygenases: structural and functional insights. Journal of Lipid Research 50 (Suppl) S29-S34. (doi:10.1194/jlr. R800042-JLR200)

Sachse F, Becker K, Basel TJ, Weiss D \& Rudack C 2011 IKK-2 inhibitor TPCA-1 represses nasal epithelial inflammation in vitro. Rhinology 49 168-173. (doi:10.4193/Rhino10.099)

Shahin AY, Hassanin IM, Ismail AM, Kruessel JS \& Hirchenhain J 2009 Effect of oral $\mathrm{N}$-acetyl cysteine on recurrent preterm labor following treatment for bacterial vaginosis. International Journal of Gynaecology and Obstetrics 104 44-48. (doi:10.1016/j.ijgo.2008.08.026)

Shoji T, Yoshida S, Mitsunari M, Miyake N, Tsukihara S, Iwabe T, Harada T \& Terakawa N 2007 Involvement of p38 MAP kinase in lipopolysaccharideinduced production of pro- and anti-inflammatory cytokines and prostaglandin $\mathrm{E}(2)$ in human choriodecidua. Journal of Reproductive Immunology 75 82-90. (doi:10.1016/j.jri.2007.05.002)

Tas SW, Vervoordeldonk MJ, Hajji N, May MJ, Ghosh S \& Tak PP 2006 Local treatment with the selective I $\mathrm{KB}$ kinase $\beta$ inhibitor NEMO-binding domain peptide ameliorates synovial inflammation. Arthritis Research \& Therapy 8 R86. (doi:10.1186/ar1958)

Underwood DC, Osborn RR, Kotzer CJ, Adams JL, Lee JC, Webb EF, Carpenter DC, Bochnowicz S, Thomas HC, Hay DW et al. 2000 SB 239063, a potent p38 MAP kinase inhibitor, reduces inflammatory cytokine production, airways eosinophil infiltration, and persistence. Journal of Pharmacology and Experimental Therapeutics 293 281-288.

Ward KW, Proksch JW, Azzarano LM, Salyers KL, McSurdy-Freed JE, Molnar TM, Levy MA \& Smith BR 2001 SB-239063, a potent and selective inhibitor of p38 map kinase: preclinical pharmacokinetics and species-specific reversible isomerization. Pharmaceutical Research 18 1336-1344. (doi:10.1023/A:1013002414678)

Received 8 November 2013

Accepted 12 December 2013 